PROCEEDINGS OF THE

AMERICAN MATHEMATICAL SOCIETY

Volume 134, Number 6, Pages 1647-1649

S 0002-9939(06)08367-5

Article electronically published on January 17, 2006

\title{
HOMOGENEOUS SOLUTIONS TO FULLY NONLINEAR ELLIPTIC EQUATIONS
}

\author{
NIKOLAI NADIRASHVILI AND YU YUAN
}

(Communicated by David S. Tartakoff)

\begin{abstract}
We classify homogeneous degree $d \neq 2$ solutions to fully nonlinear elliptic equations.
\end{abstract}

In this note, we show that any homogeneous degree other than 2 solution to fully nonlinear elliptic equations must be "harmonic". Consider the fully nonlinear elliptic equation $F\left(D^{2} u\right)=0$ with $\mu I \leq\left(F_{i j}\right)=\left(F_{M_{i j}}(M)\right) \leq \mu^{-1} I$. Nirenberg [N] derived the a priori $C^{2, \alpha}$ estimates for the above equation in dimension 2 in the 1950s. Krylov $[\mathrm{K}$ ] and Evans [E] showed the same a priori estimates for the above equations in general dimensions under the assumption that $F$ is convex. As a modest investigation of a priori estimates for general fully nonlinear elliptic equations without the convexity condition, we study the homogeneous solutions.

Theorem 0.1. Let $u$ be a continuous in $\mathbb{R}^{n} \backslash\{0\}$ homogeneous degree $d \neq 2$ solution to the elliptic equation $F\left(D^{2} u\right)=0$ in $\mathbb{R}^{n}$ with $F \in C^{1}$. Then $u$ is harmonic in a possible new coordinate system in $\mathbb{R}^{n}$, namely

$$
\sum_{i, j=1}^{n} F_{i j}(0) D_{i j} u(x)=0
$$

Consequently, $u \equiv 0$ if $-(n-2)<d<0$ or $d$ is not an integer; otherwise, $u$ is a homogeneous harmonic polynomial with integer degree $d$.

In contrast to the variational problem, Sverák and Yan $[\mathrm{SY}]$ constructed homogeneous degree less than 1 minimizers to some strongly convex functional. Also Safonov [S] earlier constructed homogeneous order $\alpha \in(0,1)$ solutions to linear non-divergence elliptic equations with variable coefficients.

As one simple application to special Lagrangian equations $\mathrm{HL} F\left(D^{2} u\right)=$ $\sum_{i=1}^{n} \arctan \lambda_{i}-c=0$, where the $\lambda_{i}$ 's are the eigenvalues of the Hessian $D^{2} u$. It follows from our theorem that any homogeneous degree other than 2 solution must be a harmonic polynomial (and it also forces $c=0$ ).

When $d \in[0,1+\alpha(n, \mu))$, our theorem also follows from Krylov-Safonov $C^{\alpha}$ estimates (cf. [CC, Corollary 5.7]). The missing case $d=2$ is delicate. One only knows that any homogeneous degree 2 solution to the above fully nonlinear elliptic equation in dimension 3 is quadratic [HNY] p. 426].

Received by the editors November 5, 2004.

2000 Mathematics Subject Classification. Primary 35J60.

Both authors were partially supported by NSF grants, and the second author was also supported by a Sloan Research Fellowship.

(C)2006 American Mathematical Society Reverts to public domain 28 years from publication 1647 
Now we show our theorem.

Proof. We first consider the case that $u$ is smooth in $\mathbb{R}^{n} \backslash\{0\}$. Set

$$
\Sigma=\left\{|x|^{d-2} D^{2} u\left(\frac{x}{|x|}\right) \mid x \in \mathbb{R}^{n} \backslash\{0\}\right\}
$$

and $\Gamma=\{M \mid F(M)=0\}$. For the homogeneous order $d$ function $u(x), D^{2} u(x)=$ $|x|^{d-2} D^{2} u\left(\frac{x}{|x|}\right)$. Let $|x| \rightarrow 0$ for $d>2$ or $|x| \rightarrow \infty$ for $d<2$; we see that $0 \in \Sigma$. Also $u$ is a solution to $F\left(D^{2} u\right)=0$. Then the cone $\Sigma \subseteq \Gamma$. Now $F \in C^{1}$ and $\left(F_{i j}(0)\right)>0$. We know that the unique tangent plane of $\Gamma$ at 0 includes $\Sigma$. It follows that $\Sigma \perp\left(F_{i j}(0)\right)$, or

$$
\sum_{i, j=1}^{n} F_{i j}(0) D_{i j} u(x)=0 .
$$

Without loss of generality, we assume $\left(F_{i j}(0)\right)=I$ throughout the proof. Then

$$
0=\triangle u(x)=|x|^{d-2}\left[d(d+n-2) u\left(\frac{x}{|x|}\right)+\triangle_{S^{n-1}} u\left(\frac{x}{|x|}\right)\right] .
$$

The remaining conclusion of the theorem follows.

Next we show the regularity of the viscosity solution $u$ away from 0 . Set $\lambda=$ $d(d+n-2)$ and $\theta=x /|x|$. To start, we prove that $u(\theta)$ is a viscosity solution to

$$
\triangle_{S^{n-1}} u+\lambda u=0 .
$$

Let any smooth $\varphi(\theta)$ touch $u$ from above at $\theta_{0}$,

$$
\begin{aligned}
\varphi & \geq u \text { in a neighborhood of } \theta_{0}, \\
\varphi\left(\theta_{0}\right) & =u\left(\theta_{0}\right) ;
\end{aligned}
$$

then

$$
\begin{aligned}
|x|^{d} \varphi\left(\frac{x}{|x|}\right) & \geq|x|^{d} u\left(\frac{x}{|x|}\right) \text { in a neighborhood of } \theta_{0}, \\
|x|^{d} \varphi\left(\theta_{0}\right) & =|x|^{d} u\left(\theta_{0}\right) .
\end{aligned}
$$

From our assumption that $u$ is a viscosity (sub)solution, it follows that

$$
F\left(D^{2}\left(|x|^{d} \varphi\left(\frac{x}{|x|}\right)\right)\right) \geq 0
$$

or

$$
F\left(|x|^{d-2} D_{x}^{2} \varphi(\theta)\right) \geq 0 .
$$

Let $|x| \rightarrow 0$ for $d>2$ or $|x| \rightarrow \infty$ for $d<2$ and we see that $F(0) \geq 0$. If we use the fact $u$ is also a viscosity (super)solution, we can derive that $F(0) \leq 0$. So $F(0)=0$, and

$$
\frac{F\left(t D_{x}^{2} \varphi(\theta)\right)-F(0)}{t} \geq 0 .
$$

Let $t \rightarrow 0$; we see that

or

$$
\sum_{i, j=1}^{n} F_{i j}(0) D_{i j} \varphi\left(\frac{x}{|x|}\right) \geq 0
$$

$$
\triangle_{S^{n-1}} \varphi+\lambda \varphi \geq 0 .
$$


Thus $u$ is a viscosity subsolution to (0.1). Similarly, $u$ is a viscosity supersolution to the same equation.

Let $N_{\varepsilon}$ be an $\varepsilon$ neighborhood of any $\theta_{0}$ on $S^{n-1}$, with $\varepsilon$ small enough so that $N_{\varepsilon}$ is in a narrow strip. Then there exists a positive smooth function $h$ on $N_{\varepsilon}$ such that

$$
\triangle_{S^{n-1}} h+\lambda h \leq 0 .
$$

Let $\psi$ be the smooth solution to (0.1) in $N_{\varepsilon}$ with the boundary value $u$ on $\partial N_{\varepsilon}$. Then $q=\frac{\psi-u}{h}$ is a viscosity solution to

$$
\triangle_{S^{n-1}} q+2 \frac{\nabla h}{h} \cdot \nabla q+\frac{\triangle_{S^{n-1}} h+\lambda h}{h} q=0,
$$

where $\nabla h \cdot \nabla q$ simply denotes some linear combination of first-order derivatives of $q$ in some local coordinates for $N_{\varepsilon}$, which we avoid for the sake of simple notation. Now that the coefficient $\frac{\triangle_{S n-1} h+\lambda h}{h} \leq 0$, it follows from [W] Corollary 3.20] that $q=0$ in $N_{\varepsilon}$. Therefore, $u$ is smooth in $N_{\varepsilon}$ and then on the whole $S^{n-1}$.

\section{REFERENCES}

[CC] Caffarelli, L. A. and Cabré, X., Fully nonlinear elliptic equations. American Mathematical Society Colloquium Publications, 43, American Mathematical Society, Providence, RI, 1995. MR:1351007 (96h:35046)

[E] Evans, L. C., Classical solutions of fully nonlinear, convex, second-order elliptic equations, Comm. Pure Appl. Math. 35 (1982), 333-363. MR.0649348 (83g:35038)

[HNY] Han, Q., Nadirashvili, N., and Yuan, Yu, Linearity of homogeneous order-one solutions to elliptic equations in dimension three, Comm. Pure Appl. Math. 56 (2003), 425-432. MR:1949137 (2003k:35043)

[HL] Harvey, R. and Lawson, H. B. Jr., Calibrated geometry, Acta Math. 148 (1982), 47-157. MR0666108 (85i:53058)

[K] Krylov, N. V., Boundedly nonhomogeneous elliptic and parabolic equations, Izv. Akad. Nauk SSSR Ser. Mat. 46 (1982), 487-523 in Russian; English translation in Math. USSR Izv. 20 (1983), 459-492. MR0661144 (84a:35091)

[N] Nirenberg, L., On nonlinear elliptic partial differential equations and Hölder continuity, Comm. Pure Appl. Math. 6 (1953), 103-156. MR0064986 (16:367c)

[S] Safonov, M. V., Unimprovability of estimates of Hölder constants for solutions of linear elliptic equations with measurable coefficients, (Russian) Mat. Sb. (N.S.) 132(174) (1987), no. 2, 275-288; translation in Math. USSR-Sb. 60 (1988), no. 1, 269-281. MR.0882838 (88e:35049)

[SY] Sverák, V. and Yan, X., Non-Lipschitz minimizers of smooth uniformly convex functionals, Proc. Natl. Acad. Sci. USA 99 (2002), 15269-15276. MR.1946762 (2003h:49066)

[W] Wang, L., On the regularity theory of fully nonlinear parabolic equations I, Comm. Pure Appl. Math. 45 (1992), 27-76. MR1135923 (92m:35126)

LATP, Centre de Mathématiques et Informatique, 39, Rue F. Joliot-Curie, 13453 Marseille Cedex, France

E-mail address: nicolas@cmi.univ-mrs.fr

Department of Mathematics, Box 354350, University of Washington, Seattle, WashINGTON 98195

E-mail address: yuan@math.washington.edu 